\title{
Recent Progress in the Radial Integration Boundary Integral and Integro- Differential Equation Methods for Numerical Solution of Problems with Variable Coefficients
}

\section{AL-Jawary MA ${ }^{1}$ and Wrobel LC $^{1 *}$}

\section{${ }^{1}$ School of Engineering and Design, Brunel University, Uxbridge UB8 3PH, UK}

The Boundary Element Method (BEM) has become an efficient and popular alternative to the Finite Element Method (FEM) because of its ability, at least for some problems with constant coefficients, of reducing a Boundary Value Problems (BVP) for a linear Partial Differential Equation (PDE) defined in a domain to an integral equation defined on the boundary, leading to a simplified discretisation process with boundary elements only. The main requirement for the reduction of the PDE to a Boundary Integral Equation (BIE) is that a fundamental solution to the PDE must be available. Such fundamental solutions are well known for many PDEs with constant coefficients, see [1-4], but are not generally available when the coefficients of the original PDE are variable.

BEM formulations for solving non-linear, non-homogeneous problems and problems with variable coefficients usually adopt fundamental solutions for a simplified linear, homogeneous problem with constant coefficients, generating domain integrals in the corresponding integral equation. This feature makes the BEM less attractive as a domain discretisation is then required.

Several methodologies have been proposed in order to overcome these difficulties. One possible technique is to find a fundamental solution for the non-linear, non-homogeneous problem or problem with variable coefficients which can provide a pure boundary integral equation. Unfortunately, these fundamental solutions are only available for some very special cases [5-8].

An alternative methodology for solving PDEs with variable coefficients with the BEM without domain discretisation involves the transformation of the domain integrals appearing in the integral equation, derived by using fundamental solutions for linear homogeneous problems, into equivalent boundary integrals. There are two powerful techniques available in literature: the first is the Dual Reciprocity Method (DRM) developed by Nardini and Brebbia [9]. In this method, the transformation is carried out by approximating the source term with a series of basic functions and by using their particular solutions. A detailed description and practical applications of this method can be found in the book of Partridge et al. [10]. The drawback of this technique is that the particular solutions may be difficult to obtain for some complicated problems, depending on the Radial Basis Function (RBF) adopted. In addition, even for known source terms, the method still requires an approximation of the known function using RBFs [11].

More recently, a new transformation technique, the Radial Integration Method (RIM), has been developed by Gao [11]. The RIM can transform any complicated domain integral to the boundary, while also removing various singularities appearing in the domain integrals. The main feature of the RIM is that it can treat different types of domain integrals in a unified way since it does not resort to particular solutions as in the DRM.

Another methodology for solving PDEs with variable coefficients is to use a parametrix (Levi function), which is usually available $[12,13]$. This allows a reduction of the mathematical problem to a Boundary-Domain Integral or Integro-Differential Equation (BDIE or BDIDE) [14,17-20]. AL-Jawary and Wrobel [14-16] have successfully implemented BDIE and BDIDE formulations for stationary heat transfer in isotropic materials with variable coefficients associated with Dirichlet, Neumann and mixed boundary conditions by using domain integrals. The numerical results show that high rates of convergence are obtained with mesh refinement. A pure BEM using the RIM has been derived and implemented in $[17,18]$ to solve the two-dimensional Helmholtz equation and steady state heat conduction with variable coefficients, respectively.

A BDIE and a BDIDE formulation for stationary heat transfer with variable coefficients are presented in [19] using specially constructed localized parametrices to reduce the BVP to a Localized BoundaryDomain Integral or Integro-Differential Equation (LBDIE or LBDIDE). The use of specially constructed localized parametrices leads to sparsely populated systems of linear algebraic equations.

An implementation of the LBDIE method for the numerical solution of a second-order linear elliptic PDE with variable coefficients is presented in [20], although the formulation is restricted to Neumann boundary-value problems.

Recent work by Yang and Gao [21] adopted the Green's function for the Laplace equation in deriving normalized BDIEs for time-dependent problems with varying heat conductivities. The authors argued that, unlike the standard BEM, considering the product of variable coefficients by the unknown functions as a new variable can provide accurate results. Then, the RIM is employed to convert the resulting domain integrals into equivalent boundary integrals. However, due to the way they calculated the inner radial integral, the formulations still required to calculate the integral inside the domain and is restricted to star-shaped domains.

A new type of boundary-only integral equation technique is developed for the treatment of the two-dimensional (2D) Helmholtz equation when the material parameters and wave number vary within the medium and non-homogeneous transient heat conduction problems

${ }^{*}$ Corresponding author: Wrobel LC, School of Engineering and Design, Brunel University, Uxbridge UB8 3PH, UK, E-mail: majeed078@gmail.com ; Luiz.Wrobel@brunel.ac.uk

Received July 11, 2012; Accepted July 13, 2012; Published July 17, 2012

Citation: AL-Jawary MA, Wrobel LC (2012) Recent Progress in the Radial Integration Boundary Integral and Integro-Differential Equation Methods for Numerical Solution of Problems with Variable Coefficients. J Applied Computat Mathemat 1:e115. doi:10.4172/2168-9679.1000e115

Copyright: ( 2012 AL-Jawary MA, et al. This is an open-access article distributed under the terms of the Creative Commons Attribution License, which permits unrestricted use, distribution, and reproduction in any medium, provided the original author and source are credited. 
Citation: AL-Jawary MA, Wrobel LC (2012) Recent Progress in the Radial Integration Boundary Integral and Integro-Differential Equation Methods for Numerical Solution of Problems with Variable Coefficients. J Applied Computat Mathemat 1:e115. doi:10.4172/2168-9679.1000e115

Page 2 of 2

with variable coefficients based on the use of a parametrix [17,22]. The RIM is used to convert the domain integrals appearing in both BDIE and BDIDE to equivalent boundary integrals. Unlike the RIM available in the literature [21], in which the normalized temperature was used to formulate the integral equation, we adopt in our work the standard BDIE and new BDIDE methods to formulate the integral equation. Moreover, the radial integral is calculated only along the boundary. For domain integral consisting of known functions the transformation is straightforward, while for domain integrals that include unknown variables the transformation is accomplished with the use of augmented RBFs, similar to the DRM. The most attractive feature of the method is that the transformations are very simple and have similar forms for both two- and three-dimensional problems.

\section{References}

1. Ang WT (2001) A Beginners Course in Boundary Element Methods, Universal Publishers, Boca Raton, USA

2. LC Wrobel (2002) The Boundary Element Method, Vol.1, Chichester: Wiley.

3. Brebbia CA, Telles JCF, Wrobel LC (1984) Boundary element techniques: Theory and applications in engineering. Springer, New York, USA.

4. Paris F, Canas J (1997) Boundary Element Method Fundamentals and Applications, New York: Oxford University Press.

5. Clements DL (1998) Fundamental solutions for second order linear elliptic partial differential equations, Comp Mech 22: 26-31.

6. Ang WT, Kusuma J, Clements DL (1996) A boundary element method for a second order elliptic partial differential equation with variable coefficients. Eng Anal Bound Elem 18: 311-316.

7. RP Shaw (1994) Green's functions for heterogeneous media potential problems. Eng Anal Bound Elem 13: 219-221.

8. Shaw RP, Gipson GS (1995) A BIE formulation of a linearly layered potential problem. Eng Anal Bound Elem 16: 1-3.

9. Nardini D, Brebbia CA (1982) A new approach for free vibration analysis using boundary elements. Boundary Element Methods in Engineering, Brebbia CA Springer: Berlin 312-326.

10. Partridge PW, Brebbia CA, Wrobel LC (1992) The dual reciprocity boundary element method. Computational Mechanics Publication, Southampton.

11. Gao XW (2002) The radial integration method for evaluation of domain integrals with boundary-only discretization. Eng Anal Bound Elem 26: 905-916.

12. Venturini WS (1989) Further develpments of boundary element formulation for zoned domain problems. Advances in Boundary Elements, Computational Mechanics Publications, Southampton and Springer-Verlag, Berlin and New York.

13. Brunton I (1996) Solving variable coefficient partial differential equations using the boundary element method. University of Auckland, New Zealand.

14. AL-Jawary MA, Wrobel LC (2011) Numerical solution of two-dimensional mixed problems with variable coefficients by the boundary-domain integral and integro-differential equation methods. Eng Anal Bound Elem 35: 1279-1287.

15. AL-Jawary MA, Wrobel LC, Maischak M (2011) Numerical solution of a Dirichlet problem with variable coefficients by the boundary-domain integro-differential equation method. Eighth UK Conference on Boundary Integral Methods, University of Leeds Press, Leeds.

16. AL-Jawary MA, Wrobel LC, Maischak M (2011) Numerical solution of a Neumann problem with variable coefficients by the boundary-domain integral equation method. Eighth UK Conference on Boundary Integral Methods, University of Leeds Press, Leeds.

17. AL-Jawary MA, Wrobel LC (2012) Numerical solution of the two-dimensional Helmholtz equation with variable coefficients by the radial integration boundary integral and integro-differential equation methods. Int J Com Math 89: 14631487.

18. AL-Jawary MA, Wrobel LC (2012) Radial integration boundary integral and integro-differential equation methods for two-dimensional heat conduction problems with variable coefficients. Eng Anal Bound Elem 36: 685-695.

19. Mikhailov SE (2002) Localized boundary-domain integral formulations for problems with variable coefficients. Eng Anal Bound Elem 26: 681-690.

20. Mikhailov SE, Nakhova IS (2005) Mesh-based numerical implementation of the localized boundarydomain integral equation method to a variable-coefficient Neumann problem. J Eng Math 51: 251-259.

21. Yang K, Gao XW (2010) Radial integration BEM for transient heat conduction problems. Eng Anal Bound Elem 34: 557-563.

22. AL-Jawary MA, Ravnik J, Wrobel LC, Skerget L (2012) Boundary element formulations for numerical solution of two-dimensional diffusion problems with variable coefficients. Computers and Mathematics with Applications (In review). 